\title{
TESTE DE HODGE MODIFICADO EM ÁGAR CLED PARA TRIAGEM DE Proteus mirabilis PRODUTORES DE CARBAPENEMASE
}

\section{MODIFIED HODGE TEST WITH CLED AGAR FOR SCREENING CARBAPENEMASE- PRODUCING Proteus mirabilis}

\author{
Gabrielli Stefaninni Santiago ${ }^{1}$ \\ Laura Ribeiro ${ }^{2}$ \\ Irene da Silva Coelho ${ }^{3}$ \\ Miliane Moreira Soares de Souza ${ }^{4}$ \\ Shana de Mattos de Oliveira Coelho ${ }^{5}$
}

\begin{abstract}
Resumo: Enterobacteriaceae produtoras de carbapenemase vêm sendo descritas em todo o mundo. Uma detecção precisa de bactérias produtoras de carpabenemase é necessária, pois essa classe de antibióticos é usada no tratamento de infecções severas. Em nível laboratorial, o método fenotípico para a detecção de produtores de carbapenemase é o teste de Hodge modificado. Entretanto, algumas enterobactérias têm grande motilidade, dificultando a leitura e interpretação dos resultados dessa técnica. O objetivo deste estudo foi validar um meio para se obter resultados confiáveis em bactérias com grande motilidade, como é o caso de Proteus mirabilis. O meio ágar Müller-Hinton, preconizado pelo CLSI, foi comparado ao ágar CLED que demonstrou ser um bom meio para análise da produção de carbapenemase em Proteus mirabilis suspeitos de produzirem essa enzima, embora todos os isolados tenham sido negativos no teste.

Palavras-chave: Ágar CLED; ágar Müller-Hinton; carbapenemase; Proteus mirabilis; véu de Proteus.
\end{abstract}

Abstract: Carbapenemase-producing Enterobacteriaceae have been reported worldwide. An accurate detection of bacteria producing carbapenemase is necessary since this antimicrobial class is used to treat severe infections. At a laboratory level, the standardized phenotypic method for the detection of carbapenemase production is the modified Hodge test. However, some enterobacteria have high motility, hampering the reading and interpretation of the results of this technique. The objective of this study was to validate a medium to obtain reliable results in bacteria with great motility, such as Proteus mirabilis. Compared to the medium recommended by CLSI, Müller-Hinton agar, CLED agar was observed to be a good medium when Proteus mirabilis isolates suspected of producing carbapenemase were analyzed, although it has not confirmed the production in all these isolates.

Keywords: Carbapenemase; CLED agar; Müller-Hinton agar; Proteus mirabilis; swarm.

\footnotetext{
1 Doutorado em Ciências Veterinárias, Universidade Federal Rural do Rio de Janeiro, Brasil. E-mail: gabriellissantiago@outlook.com.

2 Graduação em Medicina Veterinária pela Universidade Federal Rural do Rio de Janeiro, Brasil. E-mail: lauraribro@yahoo.com.br.

3 Doutorado em Microbiologia Agrícola, Professor adjunto na Universidade Federal Rural do Rio de Janeiro, Brasil. E-mail: irenecs@yahoo.com.

4 Doutorado em Ciências Veterinárias pela Universidade Federal Rural do Rio de Janeiro, Brasil. E-mail: milianemss@gmail.com.

5 Doutorado em Ciências Veterinárias pela Universidade Federal Rural do Rio de Janeiro, Brasil. E-mail: shana_mattos@hotmail.com.
} 


\section{INTRODUÇÃO}

Os carbapenemas são comumente usados no tratamento de infecções causadas por enterobactérias multirresistentes. O aumento de relatos de enterobactérias produtoras de carbapenemase tem causado preocupação em todo o mundo (ANDERSON et al., 2007; HIRSCH; TAM,2010; GIRLICH; POIREL; NORDMANN, 2011).

As carbapenemases têm capacidade hidrolítica versátil. Essas enzimas são hábeis em hidrolisar penicilinas, cefalosporinas, monobactâmicos e carbapenemas e, geralmente, não são inibidas pelo ácido clavulânico. KPC, VIM, IMP, NDM e OXA-48 são os principais tipos de carbapenemases (NORDMANN et al., 2012). A enzima KPC é frequentemente encontrada em Klebsiella pneumoniae e o gene codificador da enzima está presente em plasmídios transferíveis (ANDERSON et al. 2007; QUEENAN; BUSH, 2007). Esse tipo de carbapenemase também é encontrado em outras enterobactérias, como K. oxytoca, Enterobacter spp., Escherichia coli, Salmonella spp., Citrobacter freundii, Serratia spp. e Proteus mirabilis (ANDERSON et al. 2007; HIRSCH; TAM, 2010).

Proteus spp. pode ser isolado de muitos ambientes, como solo, água e trato intestinal de mamíferos e vem sendo isolado em animais de produção (WANG et al., 2014; YEH; LINE; HINTON JUNIOR, 2018). P. mirabilis tem sido descrito em muitos países como produtores de $\beta$-lactamases, especialmente de carbapenemase (BONNET et al., 2002; TIBBETTS et al., 2008; HU et al., 2012; DATTA et al., 2014).

O teste de Hodge modificado é reconhecido pelo CLSI como ferramenta fenotípica que auxilia na detecção de enterobactérias produtoras de carbapenemases $(\mathrm{HIRSCH}$; TAM, 2010; LEE et al., 2010; GIRLICH; POIREL; NORDMANN, 2011). Em algumas espécies com grande motilidade, como as do gênero Proteus, a interpretação fica prejudicada pela formação do swarm, uma deformação na área em torno do crescimento bacteriano.

Com o propósito de identificar Proteus mirabilis produtores de carbapenemase por meio do teste de Hodge modificado, foram utilizados dois meios distintos para a avaliação de quarto isolados suspeitos de produzirem essa enzima. A partir da comparação das leituras dos meios de cultura, foi possível validar um meio para detecção de carbapenemase em Proteus mirabilis e em outras espécies que, também, apresentam grande motilidade.

\section{MATERIAIS E MÉTODOS}

\subsection{Isolados bacterianos}

Foram utilizados quatro isolados de Proteus mirabilis provenientes de leite cru mastítico. Esses isolados foram previamente identificados por testes bioquímicos e 
MALDI-TOF MS, e caracterizados como resistentes a imipenem e ertapenem por difusão em disco (CLSI, 2017).

\subsection{Cepas controle}

Como controles para o teste de Hodge modificado convencional e sugerido no estudo foram utilizados: Escherichia coli ATCC25922 sensível aos $\beta$-lactâmicos; Klebsiella pneumoniae BAA1705, produtor de carbapenemase; e $K$. pneumoniae BAA1706, não produtor de carbapenemase.

\subsection{Meios e Antibióticos}

O crescimento dos isolados suspeitos de produzirem carbapenemase e as cepas controle foram cultivadas em ágar MacConkey (AMC) (Himedia). Os ajustes de turbidez foram feitos em solução salina $0,9 \%$ (Vetec). O teste de Hodge modificado foi realizado segundo o CLSI (2017) e para a realização do método convencional foi usado ágar Müller-Hinton $(\mathrm{MH})$ e para o método sugerido foi usado ágar CLED. As placas foram preparadas segundo orientações dos fabricantes (Himedia). Os discos de antibiótico utilizados para a observação da produção enzimática nos dois métodos foram do carbapenema ertapenem 10 $\mu \mathrm{g}$ (Sensidisc, DME).

\subsection{Teste de Hodge Modificado}

Uma cultura crescida por $24 \mathrm{~h}$ em ágar MacConkey da cepa controle E. coli ATCC25922 foi ajustada de acordo com a escala de turvação 0,5 de McFarland e foi inoculada com um suabe sobre a superfície da placa de CLED. O mesmo procedimento foi realizado para a inoculação da bactéria na placa de $\mathrm{MH}$. No centro da placa, foi depositado o disco de antimicrobiano ertapenem. Em seguida, os isolados Proteus mirabilis suspeitos de produzirem a enzima e os controles $K$. pneumoniae BAA1705 e BAA1706 foram semeados da borda do disco antimicrobiano até a borda da placa do meio de cultura, após terem sido ajustados segundo a escala 0,5 de McFarland. As placas foram incubadas, em estufa bacteriológica, por $24 \mathrm{~h}$ a $35^{\circ} \mathrm{C}$. O teste foi interpretado como positivo quando houve distorção da zona de inibição, se comparado com a distorção ocorrida na cepa padrão produtora de carbapenemase $K$. pneumonia BAA 1705. O teste de Hodge modificado foi realizado em triplicata para cada isolado em ágar MH e em ágar CLED (CLSI, 2017). 


\section{RESULTADOS}

Primeiramente, o teste convencional preconizado pelo CLSI (2017) foi realizado inoculando a cepa Escherichia coli ATCC25922 nas placas de ágar MH e, em seguida, os isolados Proteus mirabilis e Klebsiella pneumoniae inoculados em linhas desde o disco de ertapenem até a borda da placa. Após a incubação, observou-se que na região interna do halo formado ao redor do disco de antimicrobiano havia um sombreado característico do crescimento de Proteus spp., conhecido como "véu" ou swarm. Neste caso não foi possível realizar uma leitura confiável do teste de produção de carbapenemase nestes isolados (Figura 1).

Figura 1 - Proteus mirabilis inoculado em ágar MH. A seta aponta para o véu de Proteus que dificulta a leitura deste teste. Legenda: 1 e 2 - isolados $P$. mirabilis (provenientes do estudo de detecção de enterobactérias produtoras de $\beta$ lactamases); CN - controle negativo, Klebsiella pneumoniae BAA1706; CP controle positivo, K. pneumoniae BAA1705.

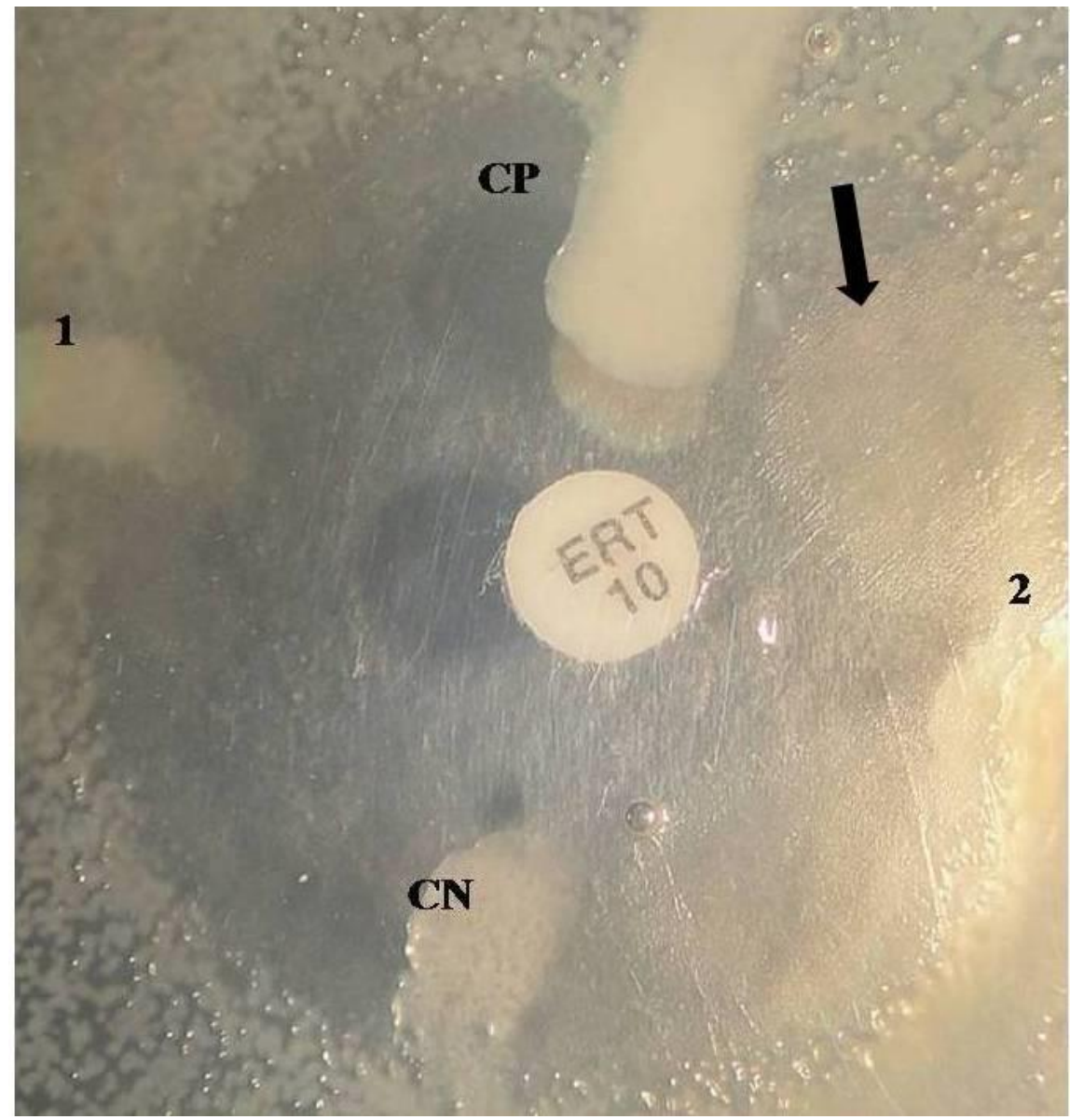

Fonte: Os autores.

No teste proposto neste trabalho, selecionou-se o meio CLED para substituição do ágar $\mathrm{MH}$ no teste de Hodge modificado, como forma de conter a motilidade do isolado 
suspeito. Assim, pode-se realizar a leitura da produção de carbapenemase nos quatro isolados de $P$. mirabilis. Comparado ao resultado apresentado pela cepa padrão $K$. pneumoniae BAA1705, não houve confirmação da produção da enzima (Figura 2).

Figura 2 - Teste de Hodge Modificado realizado em ágar CLED. A ausência do "véu" de Proteus nesse meio de cultura possibilita a leitura correta do teste: a observação da presença ou ausência da distorção no halo formado em torno do disco de ertapenem. No isolado em estudo, não foi confirmada a produção de carbapenemase, apenas a cepa padrão usada como controle positivo demonstrou a deformação no halo, característico da produção da enzima. Legenda: 3 - P. mirabilis (isolado estudado); $\mathrm{CN}$ - controle negativo, $K$. pneumoniae BAA1706; CP - controle positivo, K. pneumoniae BAA1705.

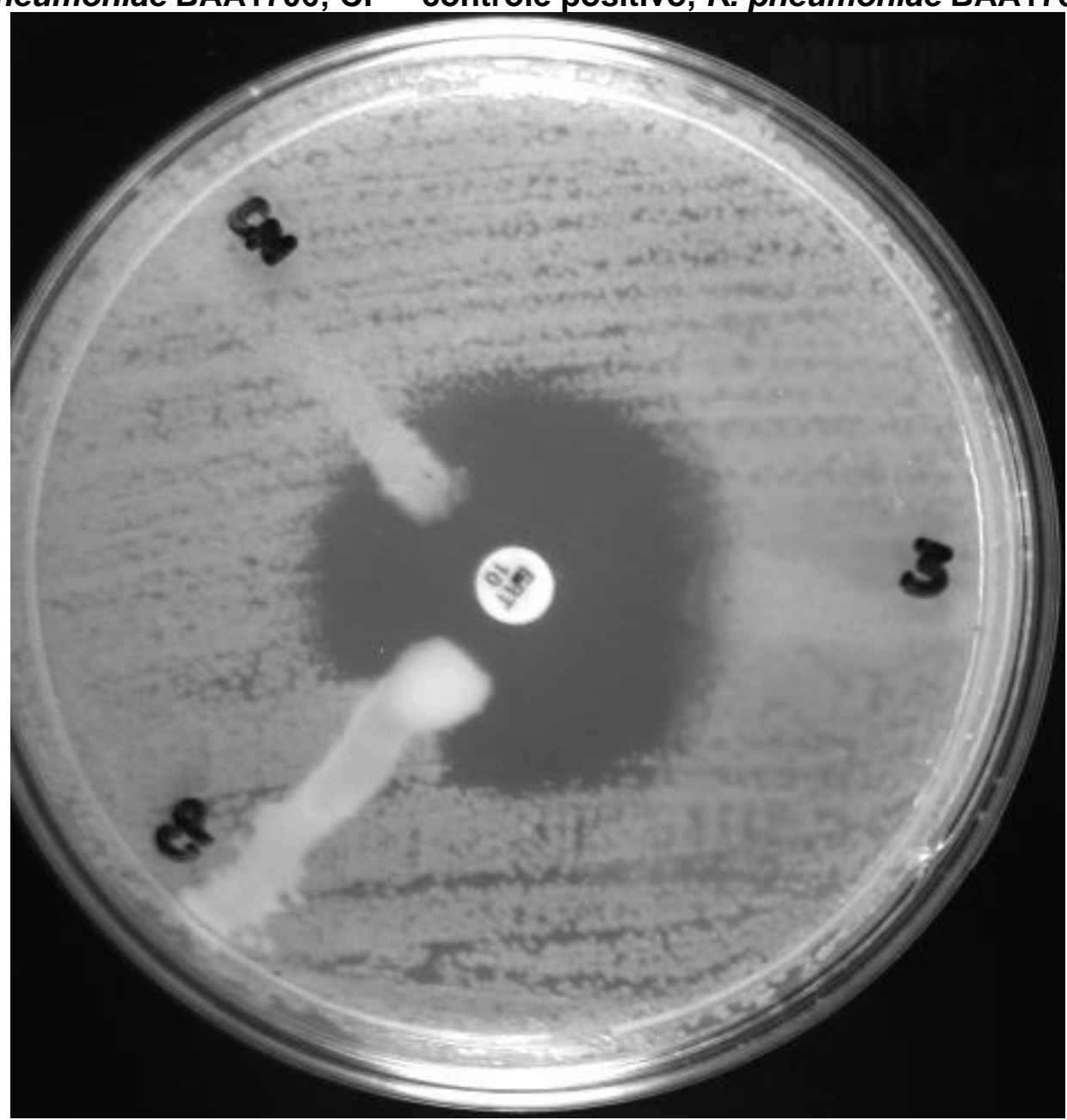

Fonte: Os autores.

\section{DISCUSSÃO}

$P$. mirabilis tem grande motilidade e forma uma nuvem sobre a placa onde é inoculado, sendo denominada "véu" ou swarm. A motilidade é devido à presença de flagelos peritríquios, aos fatores ambientais, como a presença de alguns nutrientes no meio de cultivo e à presença de lipopolissacarídeos em sua superfície celular (LIAW et al., 2003; KWIL; KAZMIERCZAK; RÓZALSKI, 2013). 
O teste de Hodge modificado tem o objetivo de auxiliar no diagnóstico de cepas produtoras de $\beta$-lactamase carbapenemases (HIRSCH; TAM, 2010; LEE et al., 2010; GIRLICH; POIREL; NORDMANN, 2011). A detecção de carbapenemase pelo teste de Hodge modificado é confirmada quando se observa o crescimento da cepa padrão sensível aos $\beta$-lactâmicos para o interior do halo de sensibilidade formado em torno do disco de ertapenem (CLSI, 2017).

Esse teste foi realizado com $\mathrm{MH}$, de acordo com o preconizado pelo CLSI, e demonstrou-se ineficiente para a análise da produção da enzima em Proteus mirabilis isolados de leite cru mastítico. Pois observou-se uma nuvem de Proteus sobre o crescimento da cepa E. coli ATCC25922, dificultando a visualização do evento.

O CLSI (2017) preconiza a utilização do ágar MH, um meio que contém: infusão de carne bovina, caseína hidrolisada e amido. Esses nutrientes são usados para o desenvolvimento bacteriano durante a realização do teste de suscetibilidade antimicrobiana. Entretanto, o ágar $\mathrm{MH}$ não tem características limitantes de motilidade, importantes para avaliação do teste em bactérias muito móveis, como é o caso da espécie estudada. Porém, outras espécies com essa característica também podem apresentar um resultado pouco confiável.

Especificamente sobre Proteus, não foi encontrado outro artigo demonstrando a mesma problemática, talvez devido à inexistência de trabalhos sobre esse comportamento em isolados provenientes de bovinos suspeitos de produzirem carbapenemases. Dessa forma, pesquisou-se um meio capaz de limitar a motilidade dessa espécie. O ágar CLED (Cystine Lactose Electrolyte Deficient medium) foi selecionado para o teste por sua composição. A deficiência em eletrólitos nesse meio limita a proliferação de Proteus spp., de acordo com estudos sobre fatores limitantes da formação de véu, e, também, não influencia o desenvolvimento bacteriano, como demonstrado pelo crescimento e presença do evento na cepa padrão positiva para o teste (WILLIAMS; SCHWARZHOFF, 1978). O meio contém todos os nutrientes necessários para o desenvolvimento bacteriano: lactose, peptona, extrato de carne, triptona e L-cistina. A lactose é uma fonte energética para organismos capazes de utilizá-la pela via fermentativa. L-cistine é adicionada como suplemento para o crescimento de coliformes que necessitam da cistina. Os demais componentes são responsáveis por suprir a necessidade de nitrogênio e carbono das bactérias. Além desses componentes, o CLED possui um indicador de $\mathrm{pH}$, nesse caso o azul de bromotimol, que diferencia as bactérias fermentadoras de lactose das que não fermentam esse açúcar (Himedia).

No meio CLED, observou-se a ausência do "véu" produzido anteriormente pelos isolados suspeitos da produção de carbapenemase. $O$ crescimento de $P$. mirabilis ocorreu apenas na linha onde foram inoculados e a leitura do teste pôde, então, ser realizada de forma segura. Não houve o crescimento da cepa controle sensível aos $\beta$ lactâmicos para o interior do halo formado em torno do ertapenem, característica de 
isolados não produtores da enzima. Tanto no meio $\mathrm{MH}$ quanto em CLED houve produção de carbapenemase pela cepa K. pneumoniae BAA 1705 e não produção pela K. pneumoniae BAA1706, usadas como controle para os dois métodos. Com isso, podese dizer que não houve influência do meio sobre a produção da enzima ou sobre a leitura do teste. A detecção de carbapenemase foi visualizada tanto no teste preconizado pelo CLSI quanto no proposto neste trabalho.

Os quatro isolados de P. mirabilis não apresentaram deformação no halo de sensibilidade, no teste realizado em CLED, demonstrando que eles não produzem carbapenemase, embora tenham apresentado resistência a carbapenemas testados em outro momento do experimento.

Outros mecanismos podem estar relacionados à resistência observada aos carbapenemas nesses isolados, como a diminuição da permeabilidade da membrana bacteriana ou a coprodução de enzimas (TSAI; WANG; HSUEH, 2015; MOHAMMED et al., 2016; DAS et al., 2017). Esses dados são fundamentais para a escolha apropriada da antibioticoterapia que será utilizada no tratamento de infecções causadas por essas bactérias e para o monitoramento das cepas de Proteus resistentes circulantes (HARADA et al., 2014).

\section{CONCLUSÃO}

Dessa forma, o teste de Hodge modificado realizado em ágar CLED mostrou-se melhor do que o teste realizado em ágar $\mathrm{MH}$ para os isolados do estudo e pode ser utilizado para a detecção de carbapenemases em outras espécies com motilidade capaz de interferir na leitura do teste. Comprovou-se, também, que não há interferência do meio na produção da enzima nem no crescimento bacteriano.

\section{AGRADECIMENTOS}

Fundação de Amparo à Pesquisa do Estado do Rio de Janeiro (FAPERJ) e Coordenação de Aperfeiçoamento de Pessoal de Nível Superior (CAPES).

\section{REFERÊNCIAS}

ANDERSON, K. F. et al. Evaluation of Methods to Identify the Klebsiella pneumoniae Carbapenemase in Enterobacteriaceae. Journal of Clinical Microbiology, p. 27232725, 2007.

BONNET, R. et al. Chromosome-Encoded Class D $\beta$-Lactamase OXA-23 in Proteus mirabilis. Antimicrobial Agents and Chemotherapy, v. 46, n. 6, p. 2004-2006, 2002. 
CLSI. Performance Standards for Antimicrobial Susceptibility Testing. 27. ed.

CLSI supplement M100. Wayne, PA: Clinical and Laboratory Standards Institute; 2017.

DAS, A. et al. Detection of emerging antibiotic resistance in bacteria isolated from subclinical mastitis in cattle in West Bengal. Veterinary World, v. 10, n. 5, p. 517-520, 2017.

DATTA, P. et al. Epidemiology of Extended-Spectrum Beta-lactamase, AmpC, and Carbapenemase Production in Proteus mirabilis. Jpn. J. Infect. Dis., n. 67, p. 44-46, 2014.

GIRLICH, D.; POIREL, L.; NORDMANN, P. Value of the Modified Hodge Test for Detection of Emerging Carbapenemases in Enterobacteriaceae. Journal of Clinical Microbiology, p. 477-479, 2011.

HARADA, K. et al. Phenotypic and molecular characterization of antimicrobial resistance in Proteus mirabilis isolates from dogs. Journal of Medical Microbiology, n. 63, p. 1561-1567, 2014.

HIRSCH, E.B; TAM, V.H. Detection and treatment options for Klebsiella pneumoniae carbapenemases (KPCs): an emerging cause of multidrug-resistant infection. Journal of Antimicrobial Chemotherapy, p. 1-7, 2010.

HU, Y.Y. et al. Emergence of $P$. mirabilis Harboring blakPC-2 and $q n r D$ in a Chinese Hospital. Antimicrobial Agents and Chemotheraphy, p. 2278-2282. 2012.

KWIL, I.; KAZMIERCZAK, D.; RÓZALSKI, A. Swarming growth and resistance of Proteus penneri and Proteus vulgaris strains to normal human serum. Advances in Clinical and Experimental Medicine, n. 22, v. 2, p. 165-175. 2013.

LEE, K. et al. Modified Hodge and EDTA-disk sinergy tests to screen metallo-betalactamase-producing strains of Pseudomonas and Acinetobacter species. European Society of Clinical Microbiology and Infectious Diseases, p. 7, p. 88-102. 2000.

LEE, K. et al. Improved performance of the modified Hodge test with MacConkey agar for screening carbapenemase-producing Gram-negative bacilli. Journal of Microbiological Methods, n. 83, p. 149-152, 2010.

LIAW, S.J. et al. Role of RsmA in the regulation of swarming motility and virulence factor expression in Proteus mirabilis. Journal of Medical Microbiology, n. 52, p. 1928.

MOHAMMED, A. N. et al. Ecological study on antimicrobial-resistant zoonotic bacteria transmitted by flies in cattle farms. Parasitology Research n. 115, v. 10, p. 38893896, 2016.

NORDMANN, P. et al. European Network on Carbapenemases Identification and screening of carbapenemase-producing Enterobacteriaceae. European Society of Clinical Microbioloy and Infectious Diseases, n. 18, p. 432-438, 2012.

QUEENAN, A.M.; BUSH, K. Carbapenemases: the Versatile Beta-lactamases. Clinical Microbiology Reviews, n. 20, v. 3, p. 440-458, 2007. 
TIBBETTS, R. et al. Detection of KPC-2 in a Clinical Isolate of Proteus mirabilis and First Reported Description of Carbapenemase Resistance Caused by a KPC BetaLactamase in P. mirabilis. Journal of Clinical Microbiology, n. 46, v. 9, p. 3080-3083, 2008.

TSAI, Y. L.; WANG, M. C.; HSUEH, P. R. et al. Overexpression of an outer membrane protein associated with decreased susceptibility to carbapenems in Proteus mirabilis.

PLoS One, n.10, v.3, e0120395. 2015.

YEH, H-Y.; LINE, J. E.; HINTON JUNIOR, A. Molecular analysis, biochemical characterization, antimicrobial activity, and immunological analysis of Proteus mirabilis isolates from broilers. Journal of Food Science, v. 83, n. 3, p. 770-779, 2018.

WANG, J.T. et al. TSAR Hospitals. Antimicrobial susceptibilities of Proteus mirabilis: a longitudinal nationwide study from the Taiwan surveillance of antimicrobial resistance (TSAR) program. BMC Infectious Diseases, n.14, p.486, 2014.

WILLIAMS, F. D.; SCHWARZHOFF, R. H. Nature of Swarming Phenomenon in Proteus. Annual Review of Microbiology, n. 32, p. 101-122, 1978. 